\title{
Monkeys genetically modified to show autism symptoms
}

\section{But it is unclear how well the results match the condition in humans.}

\section{BY DAVID CYRANOSKI}

$\mathrm{T}$ The laboratory monkeys run obsessively in circles, largely ignore their peers and grunt anxiously when stared at. Engineered to have a gene that is related to autism spectrum disorder in people, the monkeys are the most realistic animal model of the condition yet, say their creators. Researchers hope that the animals will open up new ways to test treatments and investigate the biology of autism. But the jury is still out on how well the monkeys' condition matches human autism.

Autism has a vast array of symptoms and types, and researchers think that at least 100 genes play a part. The scientists who led the latest work, which is published on 25 January in Nature (Z. Liu et al. Nature http://doi.org/ bb3k; 2016), turned to the autism-related gene MECP2: many of the symptoms of autism are found in people who have extra copies of the gene (MECP2-duplication syndrome) as well as in people who have certain mutations in this gene (Rett's syndrome). Researchers have engineered monkeys to have autism-related genes before (H. Liu et al. Cell Stem Cell 14, $323-328 ; 2014)$, but this is the first published demonstration of a link between those genes and the animals' behaviour.

Back in 2010, the team that did the latest work, led by researchers at the Chinese Academy of Sciences' Institute of Neuroscience in Shanghai, attached human MECP2 genes to a harmless virus, which they injected into the eggs of crab-eating macaque monkeys (Macaca fascicularis). The eggs were then fertilized, and the developing embryos were implanted into female monkeys. The result was 8 genetically manipulated newborns, which each had 1-7 extra copies of $M E C P 2$. Examinations of other, stillborn monkeys revealed that the extra copies were being expressed in the brain. "That was the first exciting moment," says Zilong Qiu, a molecular biologist at the Institute of Neuroscience and a co-author of the paper.

The next breakthrough came about a year later, when the monkeys showed behaviours that hinted at autism: running around in tight circles in a strange manner. "If another monkey is in its way, it will either jump over the monkey, or go around it, but then it would return to its original circular path," says co-author Sun Qiang, a reproductive biologist at the institute.

The team launched a battery of behavioural

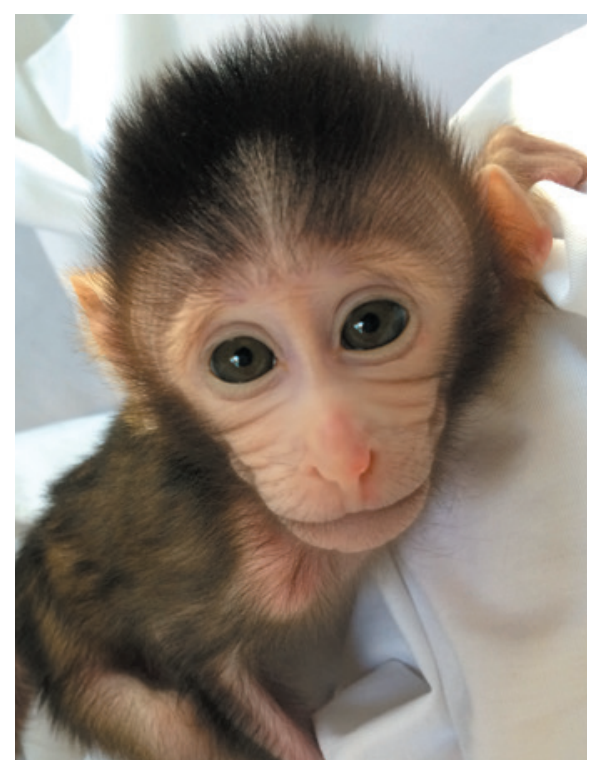

A macaque made to have autism-like behaviours.

tests, which showed that all of the monkeys had at least one autism-like symptom, such as repetitive or asocial behaviour, and that the symptoms were more severe in males, as seen in people with the MECP 2 duplications. But this still wasn't enough to be sure that the monkeys were a sound model of autism - and a paper that the team submitted for publication in 2013 was rejected. Among other things, reviewers wanted to know whether the unusual behaviour was just the result of fiddling around with the genome. "We needed to show where the gene makes a difference," says Qiu.

That opportunity came with the next generation of macaques, which the team created with unprecedented speed. When the monkeys were 27 months old and not yet sexually mature, Sun's team took testes from the males, matured the tissue artificially by grafting it under the skin on the backs of castrated mice, and used the resulting sperm to fertilize eggs from nonengineered macaques. The offspring showed asocial behaviour at about 11 months. That both gene and symptoms seemed to be passed on to a second generation was finally enough to convince reviewers, says Qiu.

The macaque model is "superior" to mouse models of autism because "it actually shows more clearly some of the autism-like behaviours", says Alysson Muotri, who researches stem cells, autism and Rett's syndrome at the

University of California, San Diego. But he adds that the symptoms in both mice and monkeys still seem less severe than "what we actually observe in human patients". "It remains to be seen if the model can actually generate novel insights into the human condition," he says.

Huda Zoghbi, a pioneer of MECP2 studies in mice at Baylor College of Medicine in Houston, Texas, is even more cautious. The monkeys do not mimic some of the human MECP2-duplication symptoms, such as seizures and severe cognitive problems, she notes. This could be because the expression of the gene in the monkey model is triggered by a different mechanism from that in humans - a limitation that the authors recognize - and she advises caution in using the model to make assumptions about human autism.

Qiu, meanwhile, is excited by the prospect of using the model to identify exactly where in the brain the MECP2 overexpression causes trouble. His team is already using brain-imaging technology on the monkeys to pinpoint such areas. Next, the researchers plan to use the CRISPR gene-editing technique to knock out the extra $M E C P 2$ copies in cells in those regions and then check whether the autisim-like symptoms stop.

It is unlikely that such a technique would be approved for use in people any time soon. But the regions identified in the monkey study could be targeted with other, existing treatments - such as deep brain stimulation, which has

"We needed to show where the gene makes a difference." of the human brain, Qiu says that the monkey imaging will allow more parallels to be drawn with humans than mice studies could. Working with a mental-health hospital, the team is also trying to identify the autism-linked genes that are most common in the Chinese population.

If non-human primates prove to be a useful model for psychiatric disorders, China and other countries that are investing heavily in research on monkeys, such as Japan, could gain an edge in brain research. Muotri says that such studies probably wouldn't be done in the United States, where research on monkeys is more expensive and controversial. "China and Japan have a clear advantage over the US on this area," he says. $\square$ 